\title{
Pleurotus Ostreatus $\beta$-glucan Alleviates \\ Cyclophosphamide-induced Immunosuppression by Regulating Gut Microbiota in Mice
}

Yalu Shao ( $\nabla$ yalu_s@aol.com )

Wuhan University

Hua Zhong

Wuhan University

Alejandro Pérez-Ponce

Hohai University

Deli Chen

The University of Melbourne

Lei Zhong

Nanjing Agricultural University

\section{Research}

Keywords: Immunosuppression, gut microbial dysbiosis, Pleurotus ostreatus $\beta$-glucan, cyclophosphamide, intestinal mucosal barrier

Posted Date: September 17th, 2020

DOI: https://doi.org/10.21203/rs.3.rs-76694/v1

License: (c) (1) This work is licensed under a Creative Commons Attribution 4.0 International License. Read Full License 


\section{Abstract}

\section{Background}

Immunosuppression has given a serious threat to human health and can induce gut microbial dysbiosis. Pleurotus ostreatus (P. ostreatus) $\beta$-glucan (POG) with an average molecular weight of $214.5 \mathrm{kDa}$, mainly consisting of $95.72 \mathrm{~mol} \%$ glucose by $\beta-(1 \rightarrow 3)$ linkages, can probably act as promising probiotics to modulate gut microbiota for relieving immunosuppression.

Results

In this study, based on a mouse model of cyclophosphamide-induced immunosuppression, we found that high dose of POG (HPOG, $0.9 \mathrm{~g} / \mathrm{kg}$ bodyweight) could stimulate immune response by increasing the concentrations of cytokines and immunoglobulins in serum. After administration of POG, the pathological damages to spleen and thymus were recovered in immunosuppressed mice, accompanied with an improved intestinal mucosal barrier maintained by tight junction proteins. Additionally, HPOG enriched short chain fatty acids and restored gut microbiota composition in immunosuppressed mice. Specifically, HPOG-mediated genera, including Intestinimonas (OTU185, OTU232), Bacteroides (OTU002, OTU034) and Barnesiella (OTU046), were found to have significant Spearman's correlation with the recovery of immune function and intestinal mucosal barrier.

Conclusions

These findings suggested that POG had the potential to protect mice from immunosuppression by "drugging the gut microbiota".

\section{Introduction}

Intestinal mucosal barrier, such as chemical and biological barriers, is the first line of defense against invasive pathogens and plays a critical role in intestinal homeostasis [1]. The damage to intestinal mucosal barrier can cause the reduced secretion of immunoglobulins and impaired function of immune organs, thereby diminishing an immune response [2]. The gut microbiota, a complex community consisting of over 100 trillion bacteria in the distal gut, and their metabolites constitute the chemical and biological barriers of intestinal mucosa [3]. The previous report indicated that specific bacterial phylotypes were parallel to the expression of tight junction proteins, but impaired intestinal mucosal barrier could induce bacterial translocation [4]. Thus, the healthy gut microbiota composition can make huge contributions to the regulation of intestinal mucosal barrier dysfunction and immune system disorders.

Immunosuppression is a distinctive feature of immune system disorders, manifesting as a destroyed balance of immune function [5]. Cyclophosphamide (CTX), a potent alkylating agent in immunosuppression, has been found to induce gut microbial dysbiosis, as characterized by an increased 
abundance of pathogenic bacteria, the decreased bacterial diversity and bacterial load [6]. Han et al. (2020) found that CTX could destroy intercellular space of intestinal epithelium in mice, manifesting as the down-regulation of zonula occludins-1 (ZO-1), Claudins-1 and Occludin [7]. This finding suggested that CTX-induced immunosuppressed mice were accompanied with damaged integrity of intestinal mucosal barrier and gut microbial dysbiosis. Recently, numerous evidences indicated that hostindigestible polysaccharides could pass through the stomach and enter the intestine in the form of original state or partially hydrolyzed fragments [8]. During intestinal absorption, gut microbiota can enrich short chain fatty acids (SCFAs) by metabolizing polysaccharides for providing direct energy to intestinal epithelial cells [9]. In turn, indigestible polysaccharides-mediated bacterial metabolism further prevents pathogenic bacteria from reproducing and stimulates the proliferation of beneficial microorganisms [1]. Thus, indigestible polysaccharides could be fully absorbed by intestine to effectively and reproducibly correct the unbalanced gut microbiota, thereby alleviating immunosuppression and intestinal mucosal barrier injury.

Pleurotus ostreatus (P. ostreatus) is a globally cultivated edible mushroom and its market share in China has been beyond over $94 \%$ market share of the total world production [10]. $\beta$-Glucan is one of the abundant components in $P$. ostreatus and exhibited multiple bioactivates, such as anti-tumor, antiinflammation and antioxidation [11-13]. It has been reported that $P$. ostreatus $\beta$-glucan (POG) can enhance an immune response by stimulating the production of superoxide anion in HL-60 cells [14]. An in vivo study showed that supplementation with POG could alleviate intensive exercise-induced immunosuppression of athletes by delaying the reduction in the activity and count of natural killer cell [15].Additionally, POG can be a potential candidate for indigestible polysaccharides to resist acidolysis and enzymolysis during the gastric phase of digestion [8]. Therefore, we hypothesized that POG could relive CTX-induced immunosuppression by modulating gut microbiota in mice, accompanied with the repairment of intestinal mucosal barrier.

To test the hypothesis, we established a mouse model of CTX-induced immunosuppression and studied the effect of POG on immune function, including the concentration of cytokines and immunoglobulins, the repair of damaged spleen and thymus. Quantitative analysis of immunohistochemistry was used to evaluate the expression of tight junction proteins in CTX-induced immunosuppression mice. Combining with Spearman's correlation analysis, we determined the composition of gut microbiota and their metabolites level for identifying their potential role in immunoregulation.

\section{Methods}

\section{Materials and reagents}

P. ostreatus was purchased from Tianfeng Biotechnology Company (Nanjing, Jiangsu). CTX, phosphate buffered saline with Tween 20 (PBST) and 2-ethylbutyric acid with the purity of $99 \%$ were purchased from Sigma-Aldrich Corp. (St. Louis, MO). Hematoxylin and Eosin (H\&E) staining kit was purchased from 
Abcam PLC (Cambridge, UK). Other analytical reagents were purchased from Sinopharm Chemical Regent Co., Ltd (Shanghai, China).

\section{Preparation of POG}

According to the previous study [16], dried $P$. ostreatus were ground and then added to the mixture of chloroform and methanol $(2: 1, \mathrm{v} / \mathrm{v})$ for extraction at $60^{\circ} \mathrm{C}(3 \mathrm{~h}$, three times). The residuals were dried and then extracted with cold $\left(25^{\circ} \mathrm{C}\right)$ and hot $\left(100^{\circ} \mathrm{C}\right)$ deionized water in succession for $6 \mathrm{~h}$. After repeating this process for three times, the remaining residuals were extracted with $5 \%$ potassium hydroxide solution containing sodium borohydride at $100^{\circ} \mathrm{C}$ for $6 \mathrm{~h}$ (three times). The extract was evaporated and then mixed with acetic acid to adjust pH 7.0. The resulting neutral solution was dialyzed (molecular weight cut off: $2 \mathrm{kDa}$ ) against ultrapure water ( $48 \mathrm{~h}$ ) and then concentrated for further lyophilization. Freeze-thawing was used to purify the obtained crude fraction for further centrifugation at $9000 \mathrm{rpm}\left(15 \mathrm{~min}, 10^{\circ} \mathrm{C}\right)$ [17]. The resulting insoluble fraction was treated with $0.1 \mathrm{M}$ sodium hydroxide at $25^{\circ} \mathrm{C}(1 \mathrm{~h})$ for further cooling to $4^{\circ} \mathrm{C}$. After centrifugation at $9000 \mathrm{rpm}\left(10^{\circ} \mathrm{C}, 15 \mathrm{~min}\right)$, the obtained soluble fraction was mixed with acetic acid to adjust pH 7.0 for further dialysis (molecular weight cut off: $2 \mathrm{kDa}$ ) against ultrapure water $(48 \mathrm{~h})$. After that, the resulting solution was concentrated and then freeze-dried to obtain POG $(91.25 \% \pm$ $2.47 \%)$. In the previous study [18], the structure of POG has been characterized and it was consisted of $95.72 \mathrm{~mol} \%$ glucose by $\beta-(1 \rightarrow 3)$ linkages with an average molecular weight of $214.5 \mathrm{kDa}$.

\section{Animal experimentation}

Fifty mice (Balb/c, six-week old) were purchased from Yangzhou University (SCXK(Jiangsu)2017-0007) and fed in Nanjing Agricultural University [SYXK(Jiangsu)2017-0007] according to National Laboratory Animal Welfare Ethics Guide. All the mice were housed at $20 \pm 2^{\circ} \mathrm{C}$ and under a $12 \mathrm{~h}$ light-dark cycle. After accommodation for one week, mice were divided in five groups (Figure 1A), including negative control, CTX, CTX + low dose of POG (LPOG), CTX + middle dose of POG (MPOG) and CTX + high dose of POG (HPOG). On days 8-10, CTX (80 mg/kg bodyweight) was intraperitoneally injected into mice for inducing immunosuppression except the negative control group. On days 11-27, mice in the negative control and CTX groups were given normal saline by gavage. Mice in POG groups were treated with LPOG $(0.1 \mathrm{~g} / \mathrm{kg}$ bodyweight/d), MPOG (0.3 g/kg bodyweight/d) and HPOG (0.9 g/kg bodyweight/d), respectively. Additionally, changes in bodyweight of mice were recorded every day. On days 26-27, feces of mice were collected and then stored at $-80^{\circ} \mathrm{C}$ until analysis. Before the end of animal experimentation, mice were anesthetized and then their blood was collected by cardiac puncture for further centrifugation $(3500 \mathrm{rpm}$, $20 \mathrm{~min}, 4^{\circ} \mathrm{C}$ ). The resulting supernatant serum was stored at $-80^{\circ} \mathrm{C}$ for further determination. Spleen and thymus tissues were dissociated and then immediately weighed to calculate the organ index based on the formula (1). After washing with normal saline, duodenum, spleen and thymus tissues were fixed with $4 \%$ paraformaldehyde. The cecal contents were separated and then frozen in liquid nitrogen, followed by storage at $-80^{\circ} \mathrm{C}$. 


\section{Organ index $=\frac{\text { The weight of tissue }(\mathrm{mg})}{\text { Mouse bodyweight }(\mathrm{g})}$}

\section{Determination of immunoglobulins and cytokines}

ELISA kits (Thermo Fisher Scientific Inc., Waltham, MA) were used to measure the concentration of cytokines and immunoglobulin in the serum, including immunoglobulin $G(\lg G)$, immunoglobulin $A(\lg A)$, immunoglobulin M (IgM), tumor necrosis factor-a (TNF-a), interleukin-10 (IL-10), interleukin-1 $\beta$ (IL-1 $\beta$ ) and interferon- $\gamma$ (IFN- $\mathrm{Y})$.

\section{Histomorphology observation}

After being fixed for $24 \mathrm{~h}$, spleen and thymus tissues were successively processed with wash, dehydration, transparent reagent, wax-transparency, embeddedness in paraffin and then stained with Hematoxylin and Eosin (H\&E) staining [19]. The prepared slice was observed and taken photos using a microscope (Axioscope 5, Carl Zeiss AG, Oberkochen, Germany).

\section{Immunohistochemical analysis}

Duodenum tissues were embedded into paraffin and then conducted by immunohistochemical analysis to determine the expression of tight junction proteins, including ZO-1, claudin-1 and occludin. Briefly, after processing with dewaxing, rehydration and blocking, the slides were incubated with these three tight junction antibodies, respectively. After incubation for $12 \mathrm{~h}\left(4^{\circ} \mathrm{C}\right)$ and then washing with PBST, slides were further incubated with Streptavidin-HRP (Cell Signaling Technology, Inc., Danvers, MA) for $1 \mathrm{~h}$. Before immunohistochemical observation by fluorescence microscope (Zeiss Axio Observer.Z1, Zeiss, Oberkochen, Germany), slides were treated with 3,3'-Diaminobenzidine substrate (Thermo Fisher Scientific Inc., Waltham, MA) staining and then Hematoxylin. Image Pro Plus (Version 6.0, Media Cybernetics, Inc., Rockville, MD, Maryland) was used to quantify the immunohistochemistry for evaluating the expression of tight junction proteins.

\section{Gut microbiota analysis}

The extraction of fecal DNA was performed with QIAamp DNA Stool Kit (QIAGEN N.V., Hilden, Germany) for further amplifying V3-V4 regions of 16S rDNA. The sequencing of gut microbiota was conducted based on the Illumina MiSeq platform (Primer $F=$ Illumina adapter sequence 1+ CCTACGGGNGGCWGCAG, Primer $\mathrm{R}$ = Illumina adapter sequence 2+ GACTACHVGGGTATCTAATCC). After being filtered and merged, the obtained clean reads were clustered into operational taxonomic units (OTUs) at a cut-off of $97 \%$. a-Diversities and $\beta$-diversities were analysed by Mothur and $\mathrm{R}$ Language Packages (V3.4.0).

\section{Determination of SCFAs}


As previously described [8], the collected cecal contents were mixed with ultrapure water completely and then treated with vortex oscillation for $25 \mathrm{~min}$. After centrifugation at 11,000 rpm for $5 \mathrm{~min}$, the resulting supernatant was mixed with 2-ethylbutyric acid and then filtered by a $0.45 \mu \mathrm{m}$ syringe filter. The concentrations of SCFAs, including acetic, propionic, $i$-butyric, $i$-valeric $n$-butyric and $n$-valeric acids, were determined by gas chromatography (Agilent 7890, Agilent Technologies, Inc., Santa Clara, CA). The detailed procedure for determining SCFAs was shown in Figure S1 of Supporting Information.

\section{Statistical analysis}

The results of gut microbiota were expressed as mean \pm standard error of measurement (SEM) and their differences among groups were assessed by the one-way analysis of variance, followed by Tukey's post hoc test. Other data were expressed as mean \pm standard deviation (SD) and their differences among groups were assessed by the one-way analysis of variance, followed by Duncan's multiple-range test. The data analysis was performed with SPSS software (24.0 version, IBM Corporation, Armonk, NY) and $p<$ 0.05 was deemed to statistical significance.

\section{Results}

\section{Effect of POG on bodyweight and organ index of CTX-induced immunosuppressed mice}

As shown in Figure 1B1, the bodyweight of mice on day 10-11 significantly $(p<0.05)$ decreased after intraperitoneal injection of CTX for three days (days 8-10). Compared with the negative control group, the bodyweight growth rate of mice was lower but that increased after treatment with HPOG. Additionally, a similar bodyweight growth rate was observed in mice of the CTX, LPOG and MPOG groups. The significant $(p<0.05)$ reductions of spleen index (Figure 1B2) and thymus index (Figure 1B3) were observed for the CTX group in comparison with those of the negative control group, which primarily confirmed the validity of immunosuppressed model. After administration with MPOG and HPOG, the spleen index and thymus index of mice significantly $(p<0.05)$ increased compared with those of the CTX group.

\section{Effect of POG on the concentrations of serum immunoglobulins and cytokines in CTX-induced immunosuppressed mice}

The secretion of serum IgA (Figure 1C1), IgM (Figure 1C2) and IgG (Figure 1C3) was inhibited in the CTX group but those could be improved in mice after treatment with HPOG. Additionally, no significant differences of three immunoglobulins levels could be observed in the LPOG group. As shown in Figure 1D1-D4, the serum concentrations of IL-10, IL-1 $\beta$, TNF- $\alpha$ and IFN- $\gamma$ in the CTX group were significantly $(p<$ 0.05 ) lower than those of the negative control group. However, mice exhibited a dose-dependent increase in the secretion of these cytokines after treatment with POG.

\section{Effect of POG on the histology of spleen and thymus in CTX-induced immunosuppressed mice}


The pathological damage to spleen and thymus tissues was evaluated by H\&E staining. Mice in the negative control group had the normal structure of spleen (Figure 2A1), manifesting as compactly arranged lymphocytes, visible lymphoid nodules, red pulp and white pulp. However, the destroyed spleen structure, reduced lymphocytes number and vague boundary between red and white pulp were observed in mice of the CTX group. These damages to spleen were relieved after treatment with HPOG, especially for the clear pulp boundary and increased lymphocytes number. As shown in Figure 2A2, mice in the CTX group exhibited the decreased number of thymocytes and disappeared boundary between thymic cortex and thymic cortex. A dose-dependent decrease in intercellular space was observed for LPOG, MPOG and HPOG groups. the CTX-induced damage to thymus tissue was recovered in HPOG groups, including compactly arranged thymocytes, which was close to the negative control group.

\section{Effect of POG on the expression of tight junction proteins in CTX-induced immunosuppressed mice}

As shown in Figure 2B1, the destroyed duodenal mucosa, atrophic villi and partially necrotic intestinal gland cells were observed in the CTX group. HPOG could recover the structure of damaged duodena in immunosuppressed mice, which was close to that of negative control. Compared with the negative control group, CTX induced the depletion of ZO-1, occludin and claudin-1 at epithelial cell junctions and significantly $(p<0.05)$ decreased the mean optical density of these three tight junction proteins (Figure $2 \mathrm{~B} 2)$. No significant $(p>0.05)$ differences in the mean optical density of ZO-1, occludin and claudin-1 were found between CTX and LPOG groups. The significant $(p<0.05)$ increase in mean optical densities of occludin and claudin-1 could be observed for MPOG groups, but HPOG could reverse changes in these three tight junction proteins. It suggested that POG could maintain the integrity of intestinal mucosa barrier by promoting the expression of ZO-1, occludin and claudin-1. The immunosuppressed mice exhibited obviously increased ZO-1, occludin and claudin-1 after treatment with HPOG.

\section{Effect of POG on the diversity of gut microbiota in CTX-induced immunosuppressed mice}

The potential immunoregulation mechanisms of POG was further identified based on gut microbiota analysis in CTX-induced immunosuppressed mice (Figure 3A). As shown in Table 1,

Table 1. Effect of POG on the a-diversity of gut microbiota in CTX-induced immunosuppressed mice

\begin{tabular}{|lllll|}
\hline & Observed OTUs & Chao- 1 & Shannon index & Simpson index \\
\hline Negative control & $463.3 \pm 13.039 \mathrm{a}$ & $534.171 \pm 20.304 \mathrm{a}$ & $4.144 \pm 0.334 \mathrm{a}$ & $0.331 \pm 0.033 \mathrm{a}$ \\
\hline CTX & $281.3 \pm 39.727 \mathrm{c}$ & $354.136 \pm 39.487 \mathrm{c}$ & $2.299 \pm 0.243 \mathrm{c}$ & $0.051 \pm 0.030 \mathrm{c}$ \\
\hline LPOG & $297.1 \pm 45.912 \mathrm{c}$ & $423.871 \pm 27.466 \mathrm{~b}$ & $3.487 \pm 0.416 \mathrm{~b}$ & $0.088 \pm 0.041 \mathrm{c}$ \\
\hline MPOG & $385.6 \pm 22.132 \mathrm{~b}$ & $434.618 \pm 31.278 \mathrm{~b}$ & $3.551 \pm 0.390 \mathrm{~b}$ & $0.190 \pm 0.101 \mathrm{~b}$ \\
\hline HPOG & $388.9 \pm 51.261 \mathrm{~b}$ & $432.718 \pm 42.264 \mathrm{~b}$ & $3.784 \pm 0.414 \mathrm{~b}$ & $0.178 \pm 0.045 \mathrm{~b}$ \\
\hline
\end{tabular}


Data were expressed as mean \pm SEM. Different letters indicated the significant $(p<0.05)$ difference among groups.

the a-diversity of gut microbiota, including observed OTUs, Chao-1, Shannon index and Simpson index, significantly $(p<0.05)$ decreased in the CTX group compared those in the negative control group. The composition of gut microbiota can be clearly clustered by principal coordinates analysis (PCoA) and cluster tree according to OTUs abundance. As shown in Figure 3B1, a separation of microflora between the negative control and the CTX group was observed along with the direction of the first principal component (PC1). Interestingly, gut microbiota of MPOG and HPOG groups are driven away from that of the CTX group and tend to that of negative control group. Additionally, the total variance of PC1 (26.14\%) was higher than that of the second principal component (PC2, 15.33\%). It indicated that MPOG and HPOG drove the gut microbiota composition disrupted by CTX towards the negative control group. Similarly, a significant divergence was further found in cluster tree (Figure 3B2).

\section{Effect of POG on gut microbiota composition at the phylum, family and OTU levels}

As shown in Figure 3C1, gut microbial phyla were mainly consisted of Bacteroidetes (Figure 3C2), Firmicutes (Figure 3C3), Proteobacteria (Figure 3C4) and their relative abundances were significantly ( $p<$ 0.05 ) altered by CTX. The ratio of Firmicutes to Bacteroidetes (Figure 3C5, F/B) increased in the CTXinduced immunosuppressed mice. Compared with the CTX group, MPOG and HPOG could significantly ( $p$ $<0.05)$ decrease the relative abundances of Firmicutes, Actinobacteria and Proteobacteria and increase the relative abundances of Bacteroidetes. However, it could be reversed in CTX-induced immunosuppressed mice after oral administration of LPOG and HPOG, suggesting that POG had the potential to relieve immunosuppression by decreasing F/B in a dose-dependent manner.

As shown in Figure 3D1, the gut microbiota were mainly consisted of six main families and they were significantly $(p<0.05)$ altered by CTX, including the decreased Porphyromonadaceae (Figure 3D2), Lactobacillaceae (Figure 3D4), Bacteroidaceae (Figure 3D6) and the increased Lachnospiraceae (Figure 3D3), Ruminococcaceae (Figure 3D5) and Desulfovibrionaceae (Figure 3D7). LPOG only altered the relative abundances of Lachnospiraceae and Lactobacillaceae in immunosuppressed mice. The relative abundance of Bacteroidaceae exhibited no significant $(p<0.05)$ difference in mice from CTX, LPOG and MPOG groups. However, these CTX-altered gut microbiota were reversed in mice after administration of POG, suggesting POG-mediated gut microbiota regulation was a dose-dependent manner.

The identification of changes in OTU-level phylotypes after treatment with POG is important because the genus of bacteria in the same family responded to CTX-induced immunosuppression. The functions of gut microbiota and their effects on immune system disorders are strain specific. Thus, OUT relative abundances over $0.1 \%$ at least in one group were chosen to analyze microbial phylotypes that were altered by CTX, LPOG, MPOG and HPOG. A total of 94 OTUs were altered CTX, and oral administration of LPOG, MPOG and HPOG changed the relative abundance of 27, 27 and 40 OTUs, respectively, leading to 59 different OTUs (Figure 3E1). Among the 59 OTUs, 36 CTX-changed OTUs were reversely changed by different doses of POG. Thereinto, 19, 19 and 25 OTUs were reversed after oral administration of LPOG, 
MPOG and HPOG, respectively, suggesting that the curative effect of POG was in a dose-dependent manner for regulating CTX-induced gut microbial dysbiosis. As shown in Figure 3E2, the relative abundances of Intestinimonas (OTU185, OTU232), Parasutterella (OTU389), Alistipes (OTU220, OTU470, OTU97), Olsenella (OTU116) and Barnesiella (OTU046, OTU80, OTU105 and OTU123) remarkably ( $p<$ 0.05) reduced in the CTX group.

\section{Effect of POG on the concentrations of SCFAs in CTX-induced immunosuppressed mice}

As shown in Figure 4, CTX could significantly $(p<0.05)$ decrease the concentration of SCFAs compared with negative control group, including acetic, propionic, $i$-butyric, $i$-valeric $n$-butyric and $n$-valeric acids. Obviously, POG could significantly $(p<0.05)$ increase the concentration of total SCFAs compared with the CTX group, resulting from the significant $(p<0.05)$ increase in acetic acid. The concentrations of propionic, $n$-butyric and $i$-valeric acids increased slightly $(p>0.05)$ in the cecal content of immunosuppressed mice after treatment with LPOG compared to those of the CTX group.

\section{Spearman's correlation of OTUs changed by POG and host phenotypes}

To ascertain specific bacteria that regulated the beneficial effects of POG on CTX-induced immunosuppression, Spearman's correlation (Figure 5) was analyzed between 59 OTUs that were altered by POG and host phenotypes. An obvious $(p<0.05)$ correlation was observed between 23 OTUs and at least one host phenotypes. Nine of 23 OTUs had a significant $(p<0.05)$ correlation with increased expression of tight junction proteins, concentrations of cytokines, immunoglobulin and SCFAs. Among 23 OTUs, LPOG, MPOG and HPOG reversed the relative abundances of 8,10 and 14 OTUs that were changed by CTX, respectively. Additionally, 5 OTUs altered by CTX were reversed by HPOG and significantly $(p<$ 0.05 ) correlated with at least one parameter of cytokines, immunoglobulin, tight junction proteins and SCFAs.

\section{Discussion}

\section{Effect of POG on the immune function in CTX-induced immunosuppressed mice}

Bodyweight and organ index are the rough reflection of body's immune function [20]. The results suggested that POG could recover the growth of spleen and thymus in CTX-induced immunosuppressed mice with a dose-dependent manner. $\lg G$, IgA, and IgM produced by differentiated $B$ cells are primarily responsible for humoral immunity [21]. HPOG could restore immune function in immunosuppressed mice. Macpherson et al. (2012) reported that gut microbiota could disseminate systemically to induce IgA and IgM response for maintaining homeostasis and preventing pathogens in the gut [22]. Additionally, it had been reported that IgG with microbial specificity played a crucial role in maintaining intestinal epithelial barrier [23]. It revealed that a potential for POG-mediated gut microbiota in resisting intestinal disorders by inducing the production of $\operatorname{IgA}$, IgG and IgM. The maintenance of homeostasis depends on these cytokines by coordinating lymphoid cells and inflammatory cells [24]. Th2 cells secrete IL-10 for promoting humoral responses, and cell-mediated immunity can be achieved by secreting IFN- $\gamma$, IL-1 $\beta$ and 
TNF-a in Th1 cells [25]. It suggested that POG had the ability to promote the proliferation and differentiation of immune cells, thereby reinforcing immunity recovery. Additionally, the synergy of different cytokines makes great contributions to the development of immune system via cell-cell communication [26]. Seo et al. (2000) showed that the anti-tumor and immunoregulation of chitosan oligomers in RAW 264.7 cells depend on TNF-a level and the synergistic effect between chitosan oligomers and IFN-Y [27]. These findings suggested that POG facilitated the release of cytokines into circulation or directly into a tissue for targeting and binding to specific immune cells, resulting in an improved immune response of CTX-treated mice. CTX has been confirmed to interfere the differentiation, development and maturation of lymphocytes in spleen and thymus, thereby inhibiting cellular and humoral immunity [28]. The observation of histopathology indicated that HPOG could protect spleen and thymus from CTX-induced damage. It was consistent with the previous report that polysaccharides derived from Cordyceps gunnii mycelia and Ganoderma atrum could protect against damages to spleen and thymus $[28,29]$.

\section{Effect of POG on the intestinal mucosal barrier in CTX-induced immunosuppressed mice}

The damage to intestinal mucosal barrier was another classic symptom of CTX-induced immunosuppression and may result in translocation of bacteria [1]. The tight junction produced the continuous barrier between intestinal epithelial cells and its integrity depended on the activation of cellular processes by tight junction proteins, such as ZOs, occludin and claudins [12]. Claudins and occludin, locating on the membrane of intestinal epithelium, can interact with ZOs to produce strong cross-links for regulating intestinal permeability [4]. The present finding indicated that POG could improve the destruction of intestinal mucosa barrier by up-regulating tight junction proteins with a dose-dependent manner.

\section{Effect of POG on the regulation of gut microbiota and SCFAs concentrations in CTX-induced immunosuppressed mice}

A defect of healthy microbiota can be observed after intraperitoneal injection of CTX [28]. POG could ameliorate the phylogenetic diversity of gut microbiota in a dose-dependent manner [30]. Our findings were consistent with the previous studies that mushroom polysaccharides shaped the overall structure of gut microbiota $[8,31]$. Similarly, CTX was reported to stimulate the proliferation of pathogens and reduction of friendly bacteria [32]. On the one hand, as the primary phyla of the gut microbiota, Bacteroidetes and Firmicutes can utilize carbohydrate active enzyme to break down carbohydrates but the degradation of polysaccharides mostly depends on Bacteroidetes [33]. Bacteroidetes were involved in the production of serval SCFAs based on glycoside hydrolases and carbohydrate metabolism [34]. It accounted for an increased Bacteroidetes and the decreased relative abundance of Firmicutes in mice after treatment with POG. On the other hand, the ratio of Firmicutes to Bacteroidetes increased in the CTXinduced immunosuppressed mice and was related to diseases, such as obesity [35], fatigue [36], metabolic disorders [37] and innate immunity [24]. Proteobacteria belongs to Gram-negative bacteria and its decreased relative abundance in the CTX group resulted from a competition between pathogenic 
bacteria and microorganisms in the intestine [38]. Additionally, symbiotic bacteria can probably utilize POG to produce SCFAs for inhibiting pathogenic bacteria [39]. Porphyromonadaceae could generate $\beta$ lactamase to promote POG degradation and its abundance was sensitive to CTX due to the immunestimulating behavior of CTX [40]. It was reported that Lactobacillaceae was correlated with the number of T lymphocytes in peripheral blood and Bacteroidaceae could make great contributions to host innate immunity [8]. Another report showed that a significantly increased Lachnospiraceae could result in colitis and immune impairment [41]. Additionally, Desulfovibrionaceae were found to be negatively related to cytokines, such as IL-2, IL-6, IL-1 $\beta$ and IFN- $\gamma$ [21]. These findings further indicated that POG was an indigestible polysaccharide and could arrive at the large intestine to regulate microbiota, which may be a potential target for CTX-induced immunoregulation. These bacteria that were found to be correlation with immunosuppression [5] and intestinal barrier damage [42] could be enriched by HPOG. In contrast, the increased bacteria in the CTX group were reduced after treatment with HPOG, including Enterorhabdus (OTU226), Parabacteroides (OTU277), Anaerotruncus (OTU295), Oscillibacter(OTU454), Allobaculum (OTU013), Bacteroides (OTU002), Odoribacter (OTU038), Bifidobacterium (OTU041), Eisenbergiella (OTU373), Turicibacter (OTU049) and Anaerobacterium (OTU130). These have been confirmed to be associated with immune regulation $[8,43]$. These findings indicated that the microbiota dysbiosis modulation could be responsible for the immunoregulatory activity of HPOG.

The metabolites of gut microbiota, especially for SCFAs, had relevance for immunosuppression pathogenesis [32]. Acetic acid was the primary SCFAs and its physiological function could be exerted after being oxidized by intestinal tract $[44,45]$. POG mostly consisting of glucose could be fermented by gut microbiota to produce propionic acid for exerting numerous biological activities, such as antiinflammatory effects, immunoregulation and intestinal barrier repair [46, 47]. Butyric acid was the major energy source of colonic mucosa and could regulate gut microbiota by inhibiting the proliferation of pathogens and increasing the growth of beneficial bacteria [48]. Additionally, it was found that SCFAs could improve the number and function of Tregs for restoring immune tolerance [30]. Therefore, the increased concentrations of SCFAs related to gut microbiota regulation could alleviate immunosuppression in mice after oral administration of POG in a dose-dependent manner. Intestinimonas (OTU185, OTU232), Bacteroides (OTU002, OTU034) and Barnesiella (OTU046), which was consistent with the previous studies $[21,49,50]$. Specifically, Bacteroides, usually colonizing in feces, has been reported to stimulate the proliferation of lymphocytes, repair dendritic cells and improve histopathological lesion of small intestine [21]. The present study demonstrated that HPOG treatment could effectively mediate CTX-induced composition of gut microbiota for improving immune response and intestinal mucosa barrier.

\section{Conclusion}

POG could enhance the immune response of CTX-induced immunosuppressed mice in a dose-dependent manner, manifesting as the high concentrations of serum cytokines (TNF-a, IL-10, IL-13, IFN- $\gamma$ ) and immunoglobulins (IgG, IgA and IgM). The improved histology of spleen and thymus was observed in immunosuppressed mice after oral administration of HPOG. Additionally, treatment with HPOG improved 
the integrity of intestinal mucosa by upregulating the expression of ZO-1, occludin and claudin-1 in mice. HPOG could modulate the diversity and composition of gut microbiota that were altered by CTX, such as Bacteroidaceae, Lactobacillaceae and Porphyromonadaceae. HPOG-mediated gut microbiota enriched the concentrations of SCFAs in immunosuppressed mice. Spearman's correlation analysis revealed that HPOG could act as the potential prebiotics for alleviating CTX-induced immunosuppression and damaged intestinal mucosa barrier by "drugging the gut microbiota" in mice.

\section{Abbreviations}

POG: Pleurotus ostreatus $\beta$-glucan; LPOG: Low dose of POG; MPOG: Middle dose of POG

HPOG: High dose of POG; CTX: Cyclophosphamide; ZO-1: Zonula occludins-1; SCFAs: Short chain fatty acids; PBST: Phosphate buffered saline with Tween 20; H\&E: Hematoxylin and Eosin; IgG:

Immunoglobulin G; IgA: Immunoglobulin A; IgM: Immunoglobulin M; TNF-a: Tumor necrosis factor-a; IL-

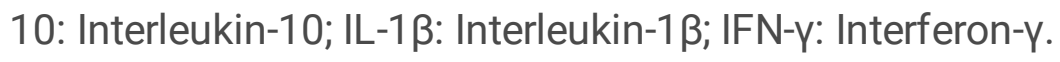

\section{Declarations}

\section{Acknowledgements}

Authors thank the staffs of Laboratory Animal Center in Nanjing Agricultural University for their help in animal experiment.

\section{Authors' contributions}

Yalu Shao designed the experiment and processed the experimental data. Hua Zhong and Alejandro Pérez-Ponce prepared the manuscript. Deli Chen and Lei Zhong conduced the experiment.

\section{Funding}

This research was funded by the Jiangsu Scientific Research Program (grant number: BE2017765); by the Fundamental Research Funds for the Central Universities (grant number: 2019B18314); by the Science and Technology Project for Nanjing Water Conservancy Bureau (grant number: 2019-208-6) and by the Undergraduate Student Research Training Program of the Ministry of Education.

\section{Availability of data and materials}

Data supporting our results are included in this article.

\section{Ethics approval and consent to participate}

Animal experiment were approved by Nanjing Agricultural University [SYXK(Jiangsu)2017-0007] according to National Laboratory Animal Welfare Ethics Guide. 


\section{Consent for publication}

Not applicable.

\section{Competing interests}

The authors declare no competing interests.

\section{References}

1. Chen D, Chen G, Ding Y, Wan P, Peng Y, Chen C, Ye H, Zeng X, Ran L. Polysaccharides from the flowers of tea (Camellia sinensis L.) modulate gut health and ameliorate cyclophosphamide-induced immunosuppression. J Funct Foods. 2019;611:03470.

2. Shi J, Zhao X-H. Chemical features of the oligochitosan-glycated caseinate digest and its enhanced protection on barrier function of the acrylamide-injured IEC-6 cells. Food Chem. 2019;290:246-254.

3. Hu B, Yu S, Shi C, Gu J, Shao Y, Chen Q, Li Y, Mezzenga R. Amyloid-polyphenol hybrid nanofilaments mitigate colitis and regulate gut microbial dysbiosis. ACS Nano. 2020;14(3):2760-2776.

4. Xiao J, Zhang R, Wu Y, Wu C, Jia X, Dong L, Liu L, Chen Y, Bai Y, Zhang M. Rice bran phenolic extract protects against alcoholic liver injury in mice by alleviating intestinal microbiota dysbiosis, barrier dysfunction, and liver inflammation mediated by the endotoxin-TLR4-Nf-kB pathway. J Agr Food Chem. 2019;68(5):1237-1247.

5. Tang C, Sun J, Zhou B, Jin C, Liu J, Kan J, Qian C, Zhang N. Effects of polysaccharides from purple sweet potatoes on immune response and gut microbiota composition in normal and cyclophosphamide treated mice. Food Funct. 2018;9(2):937-950.

6. Sun Y, Su J, Yang S, Liu Z, Liu D, Gan F, Chen X, Huang K. Mannan Oligosaccharide Protects against the Aflatoxin-B1-Promoted Influenza Replication and Tissue Damages in a Toll-Like-Receptor-4Dependent Manner. J Agr Food Chem. 2019;67(2):735-745.

7. Han X, Zhou Q, Bai B, Niu J, Yuan J, Zhang H, Jia J, Zhao W, Chen H. Dietary supplementation with polysaccharides from Ziziphus Jujuba cv. Pozao intervene immune response via regulating peripheral immunity and intestinal barrier function in cyclophosphamide-induced mice. Food Funct. 2020;11(7):5992-6006.

8. Ma G, Kimatu BM, Zhao L, Yang W, Pei F, Hu Q. In vivo fermentation of a Pleurotus eryngii polysaccharide and its effects on fecal microbiota composition and immune response. Food Funct. 2017;8(5):1810-1821.

9. Shi M, Yang Y, Hu X, Zhang Z. Effect of ultrasonic extraction conditions on antioxidative and immunomodulatory activities of a Ganoderma lucidum polysaccharide originated from fermented soybean curd residue. Food Chem. 2014;155:50-56.

10. Dicks L, Ellinger S. Effect of the intake of oyster mushrooms (Pleurotus ostreatus) on cardiometabolic parameters-A systematic review of clinical trials. Nutrients. 2020;12(4):1134. 
11. Deepalakshmi K, Sankaran M. Pleurotus ostreatus: an oyster mushroom with nutritional and medicinal properties. J Biochem Technol. 2014;5(2):718-726.

12. Du B, Lin C, Bian Z, Xu B. An insight into anti-inflammatory effects of fungal beta-glucans. Trends Food Sci Tech. 2015;41(1):49-59.

13. Khan AA, Gani A, Masoodi F, Mushtaq U, Naik AS. Structural, rheological, antioxidant, and functional properties of $\beta$-glucan extracted from edible mushrooms Agaricus bisporus, Pleurotus ostreatus and Coprinus attrimentarius. Bioact Carbohydr Diet Fibre. 2017;11:67-74.

14. Vetvicka V, Gover O, Karpovsky M, Hayby H, Danay O, Ezov N, Hadar Y, Schwartz B. Immunemodulating activities of glucans extracted from Pleurotus ostreatus and Pleurotus eryngii. J Funct Foods. 2019;54:81-91.

15. Bobovčák M, Kuniakova R, Gabriž J, Majtan J. Effect of Pleuran ( $\beta$-glucan from Pleurotus ostreatus) supplementation on cellular immune response after intensive exercise in elite athletes. Appl Physiol Nutr Me. 2010;35(6):755-762.

16. de Jesus LI, Smiderle FR, Cordeiro LM, de Freitas RA, Van Griensven LJ, lacomini M. Simple and effective purification approach to dissociate mixed water-insoluble $\alpha$-and $\beta$-D-glucans and its application on the medicinal mushroom Fomitopsis betulina. Carbohyd Polym. 2018;200:353-360.

17. Ruthes AC, Carbonero ER, Córdova MM, Baggio CH, Santos ARS, Sassaki GL, Cipriani TR, Gorin PAJ, lacomini M. Lactarius rufus $(1 \rightarrow 3),(1 \rightarrow 6)$ - $\beta$-d-glucans: structure, antinociceptive and antiinflammatory effects. Carbohyd Polym. 2013;94(1):129-136.

18. Zhong L, Ma N, Wu Y, Zhao L, Ma G, Pei F, Hu Q. Gastrointestinal fate and antioxidation of $\beta$-carotene emulsion prepared by oat protein isolate-Pleurotus ostreatus $\beta$-glucan conjugate. Carbohyd Polym. 2019;221:10-20.

19. Huang F, Wang J, Yu F, Tang Y, Ding G, Yang Z, Sun Y. Protective effect of Meretrix meretrix oligopeptides on high-fat-diet-induced non-alcoholic fatty liver disease in mice. Mar Drugs. 2018;16(2):39.

20. Zhu G, Jiang Y, Yao Y, Wu N, Luo J, Hu M, Tu Y, Xu M. Ovotransferrin ameliorates the dysbiosis of immunomodulatory function and intestinal microbiota induced by cyclophosphamide. Food Funct. 2019;10(2):1109-1122.

21. Ding Y, Yan Y, Chen D, Ran L, Mi J, Lu L, Jing B, Li X, Zeng X, Cao Y. Modulating effects of polysaccharides from the fruits of Lycium barbarum on the immune response and gut microbiota in cyclophosphamide-treated mice. Food Funct. 2019;10(6):3671-3683.

22. Macpherson AJ, Geuking MB, Slack E, Hapfelmeier S, McCoy KD. The habitat, double life, citizenship, and forgetfulness of IgA. Immunol Rev. 2012;245(1):132-146.

23. Zeng MY, Cisalpino D, Varadarajan S, Hellman J, Warren HS, Cascalho M, Inohara N, Núñez G. Gut microbiota-induced immunoglobulin $\mathrm{G}$ controls systemic infection by symbiotic bacteria and pathogens. Immunity. 2016;44(3):647-658.

24. Sun P, Wang J, Jiang Y. Effects of Enterococcus faecium (SF68) on immune function in mice. Food Chem. 2010;123(1):63-68. 
25. Chen J, Yang Z, Hu T, Yan Z, Niu T, Wang L, Cui D, Wang M. Immunomodulatory activity in vitro and in vivo of polysaccharide from Potentilla anserina. Fitoterapia. 2010;81(8):1117-1124.

26. Yu Z, Huang X, Yan C, Jin G, Liang Z. Effect of Fuzheng Jiedu granule on immunological function and level of immune-related cytokines in immune-suppressed mice. J Integr Agric. 2016;15(3):650657.

27. Seo W, Pae H, Kim N, Oh G, Park I, Kim Y, Kim Y, Lee Y, Jun C, Chung H. Synergistic cooperation between water-soluble chitosan oligomers and interferon- $\gamma$ for induction of nitric oxide synthesis and tumoricidal activity in murine peritoneal macrophages. Cancer Lett. 2000;159(2):189-195.

28. Meng M, Wang H, Li Z, Guo M, Hou L. Protective effects of polysaccharides from Cordyceps gunnii mycelia against cyclophosphamide-induced immunosuppression to TLR4/TRAF6/NF-KB signalling in BALB/c mice. Food Funct. 2019;10(6):3262-3271.

29. Li W, Li L, Zhen W, Wang L, Pan M, Lv J, Wang F, Yao Y, Nie S, Xie M. Ganoderma atrum polysaccharide ameliorates ROS generation and apoptosis in spleen and thymus of immunosuppressed mice. Food Chem Toxicol. 2017;99:199-208.

30. Ni J, Wu G, Albenberg L, Tomov V: Gut microbiota and IBD: causation or correlation? Nat Rev Gastroenterol. 2017;14(10):573.

31. Su A, Yang W, Zhao L, Pei F, Yuan B, Zhong L, Ma G, Hu Q. Flammulina velutipes polysaccharides improve scopolamine-induced learning and memory impairment in mice by modulating gut microbiota composition. Food Funct. 2018;9(3):1424-1432.

32. Shi H, Chang Y, Gao Y, Wang X, Chen X, Wang Y, Xue C, Tang Q. Dietary fucoidan of Acaudina molpadioides alters gut microbiota and mitigates intestinal mucosal injury induced by cyclophosphamide. Food Funct. 2017;8(9):3383-3393.

33. El Kaoutari A, Armougom F, Gordon JI, Raoult D, Henrissat B. The abundance and variety of carbohydrate-active enzymes in the human gut microbiota. Nat Rev Microbiol. 2013;11(7):497-504.

34. Turnbaugh PJ, Ley RE, Mahowald MA, Magrini V, Mardis ER, Gordon JI. An obesity-associated gut microbiome with increased capacity for energy harvest. Nature. 2006;444(7122):1027.

35. Chen G, Xie M, Dai Z, Wan P, Ye H, Zeng X, Sun Y. Kudingcha and fuzhuan brick tea prevent obesity and modulate gut microbiota in high-fat diet fed mice. Mol Nutr Food Res. 2018;62(6):1700485.

36. Zhong L, Ma N, Zheng H, Ma G, Zhao L, Hu Q. Tuber indicum polysaccharide relieves fatigue by regulating gut microbiota in mice. J Funct Foods. 2019;63:13580

37. Chang C, Lin C, Lu C, Martel J, Ko Y, Ojcius DM, Tseng S, Wu T, Chen Y, Young J. Ganoderma lucidum reduces obesity in mice by modulating the composition of the gut microbiota. Nat Commun. 2015;6(1):1-19.

38. Xu S, Aweya J, Li N, Deng R, Chen W, Tang J, Cheong K. Microbial catabolism of Porphyra haitanensis polysaccharides by human gut microbiota. Food Chem. 2019;289:177-186.

39. Tan H, O'Toole PW. Impact of diet on the human intestinal microbiota. Curr Opin Food Sci. 2015;2:7177. 
40. Vincent C, Miller MA, Edens TJ, Mehrotra S, Dewar K, Manges AR. Bloom and bust: intestinal microbiota dynamics in response to hospital exposures and Clostridium difficile colonization or infection. Microbiome. 2016;4(1):12.

41. Li S, Wang Z, Yang Y, Yang S, Yao C, Liu K, Cui S, Zou Q, Sun H, Guo G. Lachnospiraceae shift in the microbial community of mice faecal sample effects on water immersion restraint stress. AMB Express. 2017;7(1):82.

42. Xiao L, Chen B, Di Feng TY, Li T, Chen J. TLR4 may be involved in the regulation of colonic mucosal microbiota by Vitamin A. Front Microbiol. 2019;10:268.

43. Meng M, Wang HY, Li ZB, Guo MZ, Hou LH. Protective effects of polysaccharides from Cordyceps gunnii mycelia against cyclophosphamide-induced immunosuppression to TLR4/TRAF6/NF-kappa B signalling in BALB/c mice. Food Funct. 2019;10(6):3262-3271.

44. Lallès J-P. Microbiota-host interplay at the gut epithelial level, health and nutrition. J Anim Sci Biotechnol. 2016;7(1):66.

45. Wang J, Qin C, He T, Qiu K, Sun W, Zhang X, Jiao N, Zhu W, Yin J. Alfalfa-containing diets alter luminal microbiota structure and short chain fatty acid sensing in the caecal mucosa of pigs. $J$ Anim Sci Biotechnol. 2018;9(1):11.

46. Zhang H, Chen Y, Chen Y, Ji S, Jia P, Li Y, Wang T. Comparison of the protective effects of resveratrol and pterostilbene against intestinal damage and redox imbalance in weanling piglets. J Anim Sci Biotechnol. 2020;11:1-16.

47. Sa'ad H, Peppelenbosch MP, Roelofsen H, Vonk RJ, Venema K. Biological effects of propionic acid in humans; metabolism, potential applications and underlying mechanisms. BBA-Mol Cell Biol L. 2010;1801(11):1175-1183.

48. Kasubuchi M, Hasegawa S, Hiramatsu T, Ichimura A, Kimura I. Dietary gut microbial metabolites, short-chain fatty acids, and host metabolic regulation. Nutrients. 2015;7(4):2839-2849.

49. Cano PG, Santacruz A, Moya Á, Sanz Y. Bacteroides uniformis CECT 7771 ameliorates metabolic and immunological dysfunction in mice with high-fat-diet induced obesity. PloS one. 2012;7(7):e41079.

50. Tsuda M, Hosono A, Yanagibashi T, Hachimura S, Hirayama K, Itoh K, Takahashi K, Kaminogawa S. Prior stimulation of antigen-presenting cells with Lactobacillus regulates excessive antigen-specific cytokine responses in vitro when compared with Bacteroides. Cytotechnology. 2007;55(2-3):89-101.

\section{Figures}


A
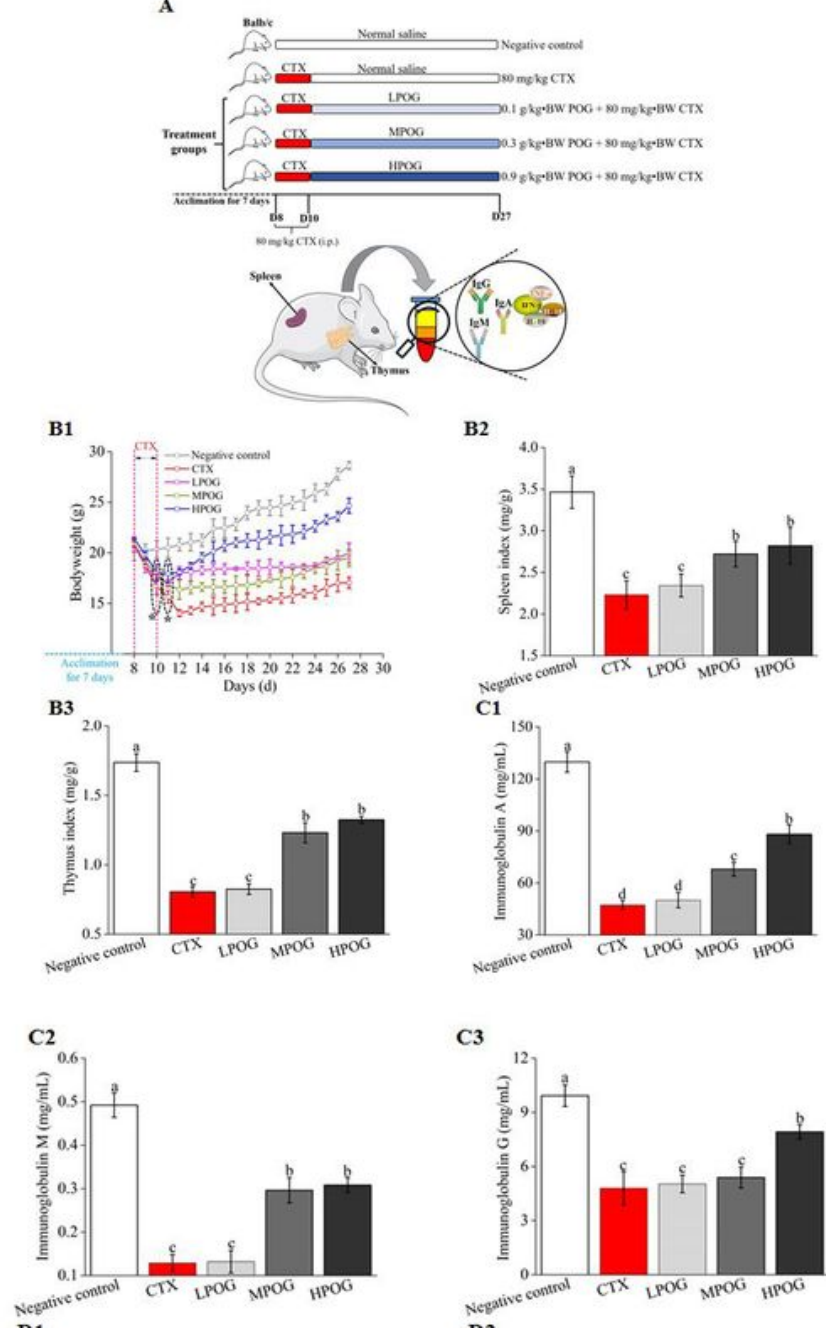

C3
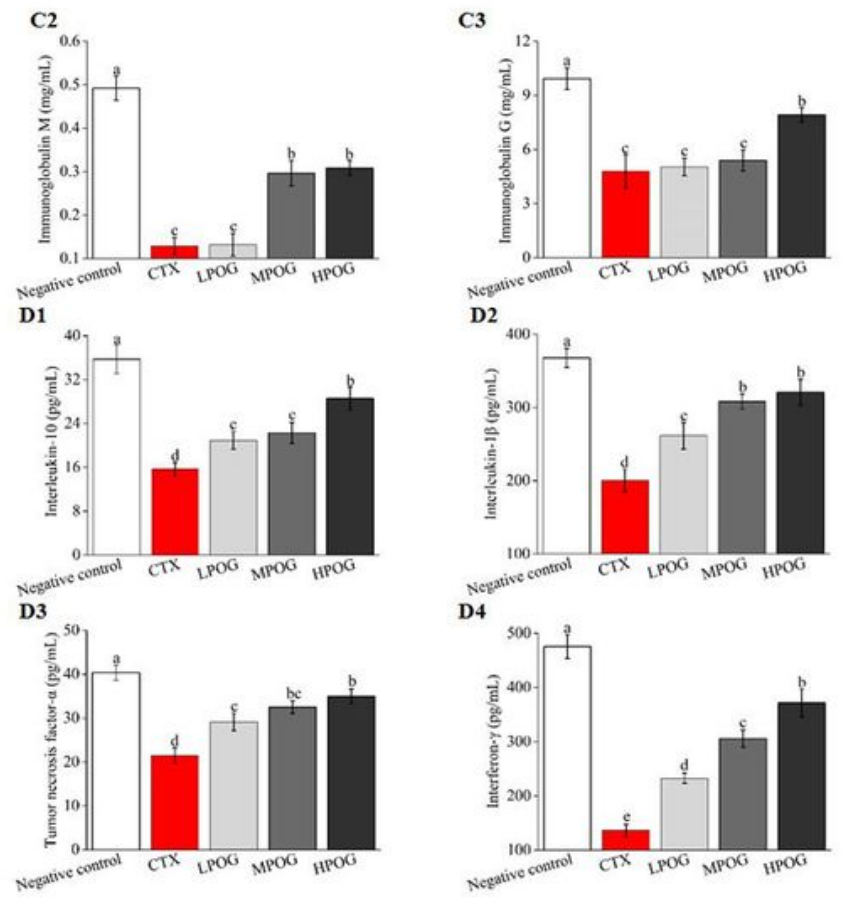

\section{Figure 1}

The flow chart of animal experimentation for 34 days (A). The figure was built based on the templates of Servier Medical Art: Smart (https://smart.servier.com/) as confirmed under a Creative Commons Attribution 3.0 Unported License; Effect of POG on the immune function in CTX-induced immunosuppressed mice, including (B1) body weight on days 8-27, spleen (B2) and thymus index (B3), immunoglobulin A (C1), immunoglobulin M (C2), immunoglobulin G (C3), interleukin-10 (D1), interleukin- 
$1 \beta$ (D2), tumor necrosis factor- $a$ (D3), interferon- $\gamma$ (D4). Data were expressed as mean \pm SD. The statistically significances $(p<0.05)$ among groups were marked with different letters. Figure 2
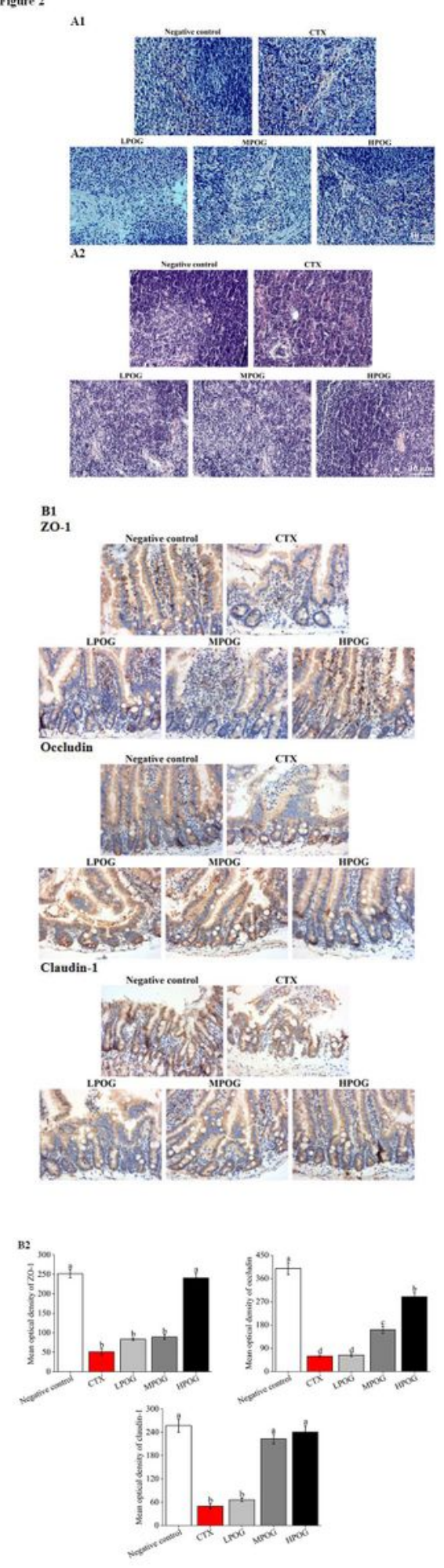

Figure 2

Histology observation for the effect of POG on restoring damaged immune organs, including spleen (A1) and thymus tissues (A2); Immunohistochemical observation for the effect of POG on repairing damaged tight junction proteins (B1); Quantitative analysis of ZO-1, occludin and claudin-1(B2). Data were 
expressed as mean \pm SD. The statistically significances $(p<0.05)$ among groups were marked with different letters.
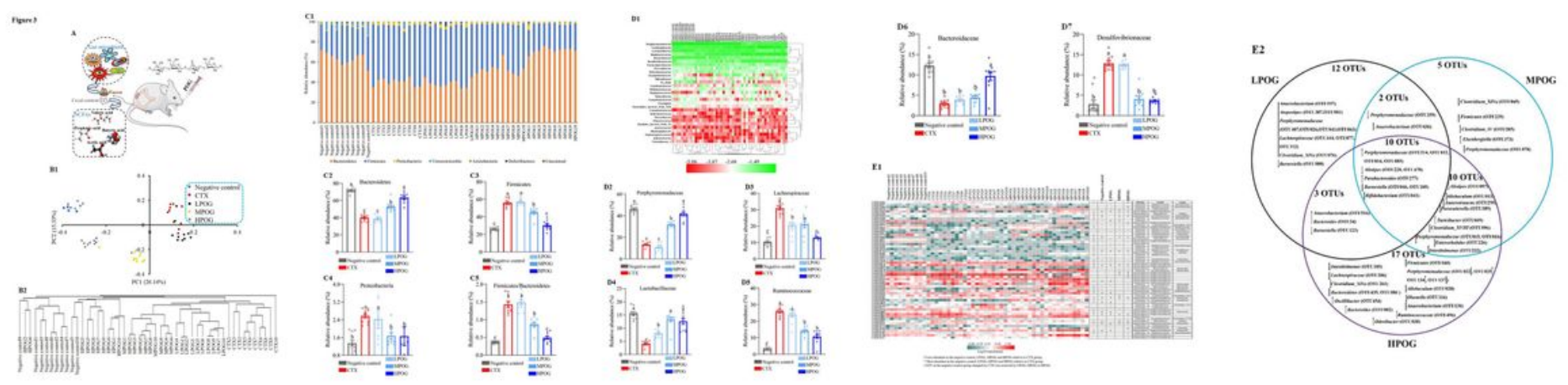

\section{Figure 3}

POG modulated the gut microbiota and SCFA levels in CTX-induced immunosuppressed mice (A). The figure was built based on the templates of Servier Medical Art: Smart (https://smart.servier.com/) as confirmed under a Creative Commons Attribution 3.0 Unported License; $\beta$-Diversity analysis, including PCoA (B1) and cluster tree based on OTU abundances (B2); The community structure analysis of gut microbial phyla (C1); The relative abundances of Bacteroidetes (C2), Firmicutes (C3), Proteobacteria (C4) and the ratio of Firmicutes to Bacteroidetes (C5); Heatmap analysis of gut microbial families (D1); The relative abundances of Porphyromonadaceae (D2), Lachnospiraceae (D3), Lactobacillaceae (D4), Ruminococcaceae (D5), Bacteroidaceae (D6) and Desulfovibrionaceae (D7). Heatmap analysis based on the relative abundances of 59 OTUs and bacterial taxonomy (phylum, family, genus) changed by LPOG, MPOG or HPOG (E1); Venn diagrams of OTUs altered by LPOG, MPOG and HPOG. $\uparrow$ means increased by POG, and $\downarrow$ means decreased by POG compared with the CTX group (E2). Data were expressed as mean \pm SEM. The statistically significances $(p<0.05)$ among groups were marked with different letters. 
Figure 4
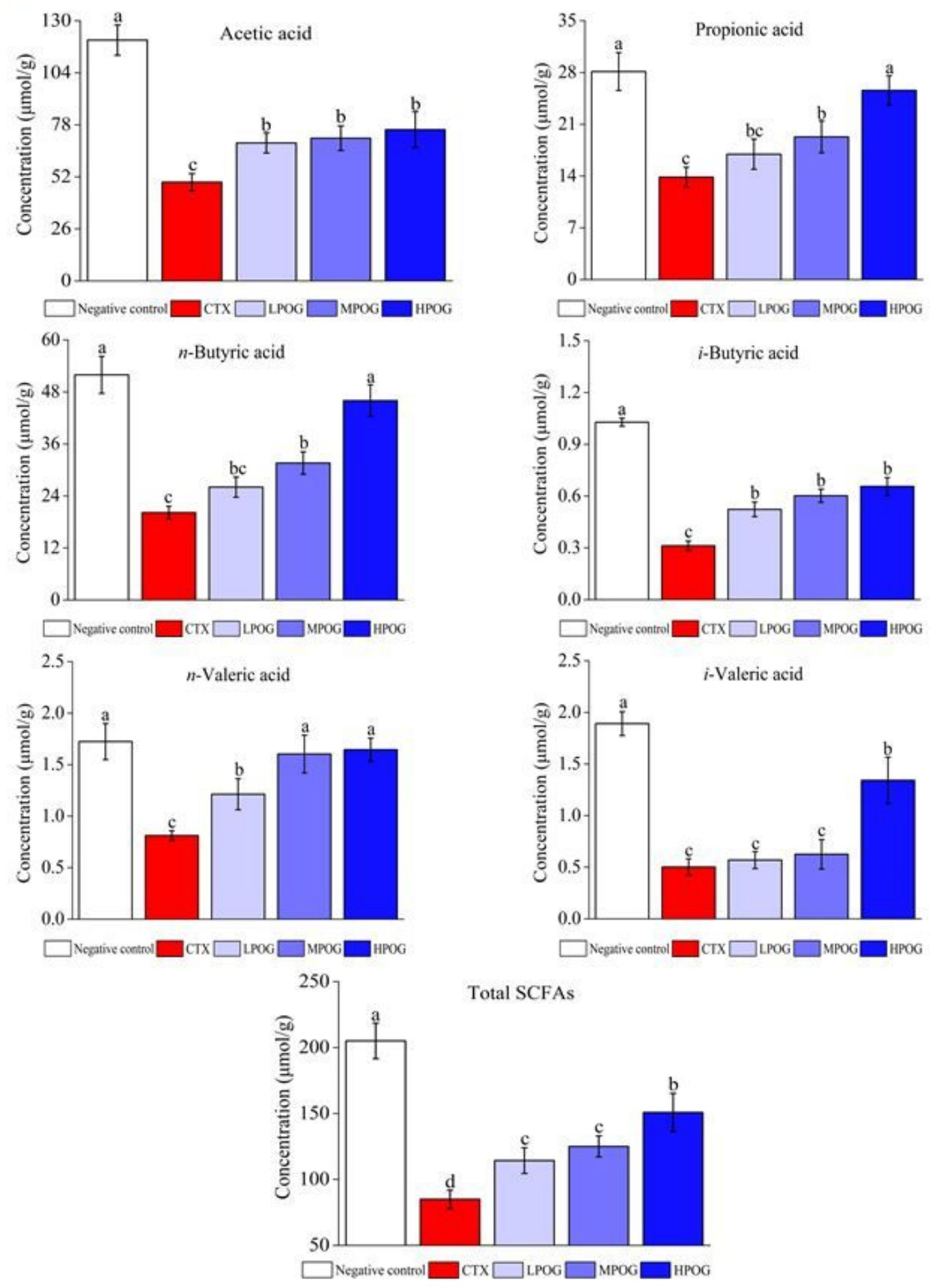

\section{Figure 4}

Effect of POG on the concentrations of SCFAs in cecal contents, including acetic, propionic, i-butyric, ivaleric, $n$-butyric and $n$-valeric acids. Data were expressed as mean \pm SD. The statistically significances $(p$ $<0.05$ ) among groups were marked with different letters. 


\section{Figure 5}

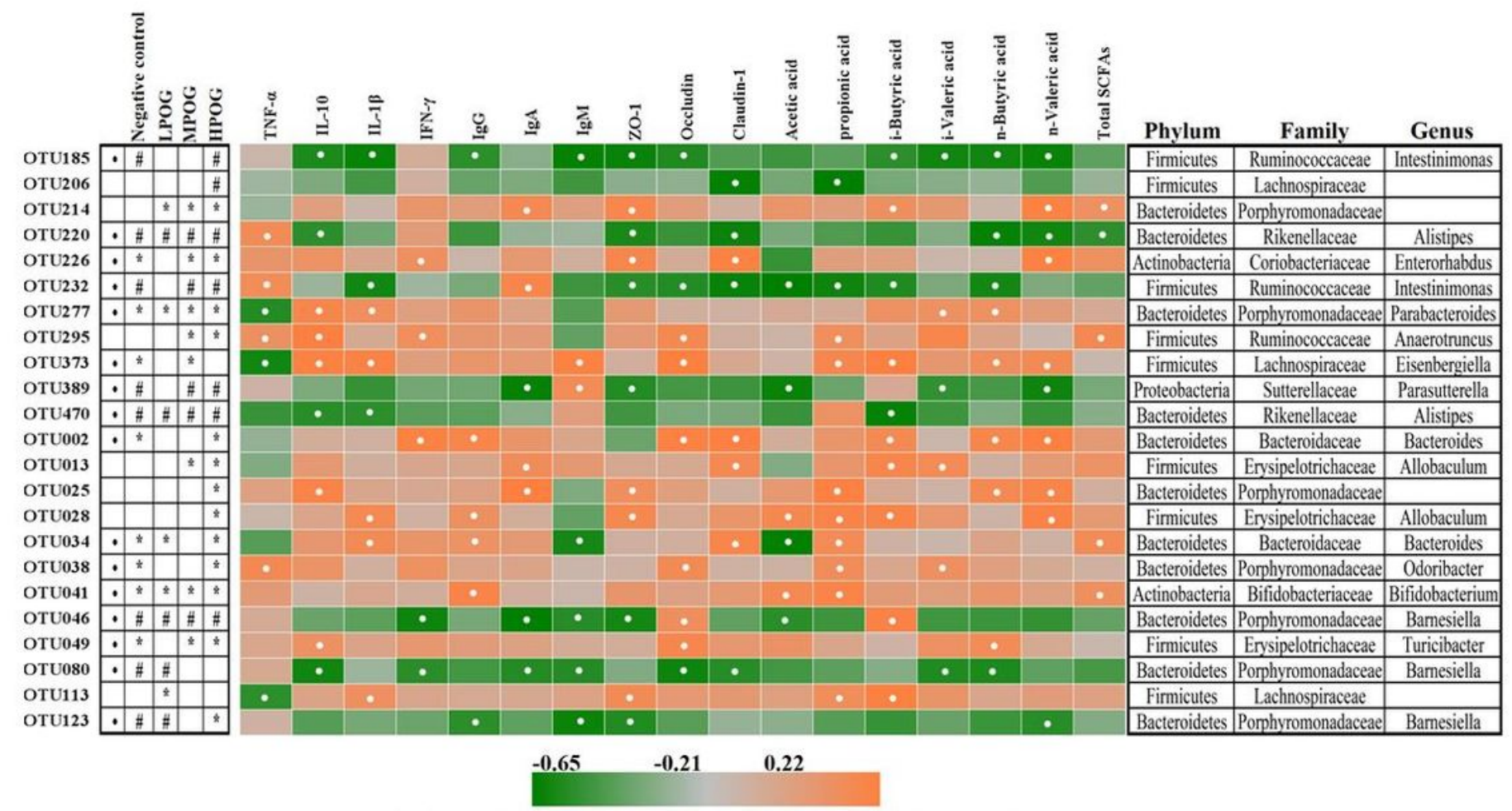

\# Less abundant in the negative control, LPOG, MPOG and HPOG relative to the CTX group.

* More abundant in the negative control, LPOG, MPOG and HPOG relative to the CTX group.

- OTU in the negative control group changed by CTX was reserved by LPOG, MPOG and HPOG.

The white dots in the cells indicate that the associations were significant (false discovery rate $<0.25$ ).

\section{Figure 5}

Spearman's correlation analysis of POG-mediated gut microbiota, immune traits, intestinal mucosa barrier and SCFAs. The statistically significant correlation was marked with white dot in the cell.

\section{Supplementary Files}

This is a list of supplementary files associated with this preprint. Click to download.

- Supplementarymaterial.docx 